\title{
ON THE HOMOTOPY GROUPS OF ONE POINT UNION WITH A BOUQUET OF CIRCLES ${ }^{1}$
}

\author{
I. BERSTEIN AND E. DROR
}

\begin{abstract}
This note points out some properties of the higher homotopy groups of $X \vee K(\pi, 1)$ as modules over $\pi$.
\end{abstract}

1. Introduction. The purpose of this note is to point out a certain property of the homotopy groups of a one-point union $X \vee S$ between a simply connected space and a one-dimensional complex $S$.

These spaces appear naturally in various contexts. For example, it is not hard to show that if

$$
L \approx S^{n} \amalg S^{n} \amalg \cdots \amalg S^{n} \subseteq S^{n+2}
$$

is any boundary link of codimension two then one can construct a map

$$
\eta:\left(S^{n+2} \backslash L\right) \rightarrow S^{n+1} \vee \cdots \vee S^{n+1} \vee S^{1} \vee \cdots \vee S^{1}
$$

such that $\eta$ induces isomorphisms on all integral homology groups. Thus the homology type of $S^{n+2} \backslash L$ does not depend on the imbedding. By boundary link one means a link whose components bound disjoint oriented submanifolds in $S^{n+2}$.

Another example of spaces of the form $X \vee S$ are all suspensions, and it is conjectured by Ganea that all co- $H$-spaces are of this type (compare [G], [B-D], [HMR]).

Let us now state our results:

THEOREM 1.1. Let $Y=X \vee K(\pi, 1)$ where $\pi$ is a torsion-free group and $X$ is simply connected. Then for all $n \geqslant 2$ the $\pi$-modules $\pi_{n}(Y, *)$ are induced i.e. $\pi_{n}(Y, *) \approx A_{n} \otimes_{\mathrm{Z}} \mathrm{Z}[\pi]$ as $\pi$-module for some abelian group $A_{n}$.

This means, roughly speaking, that the only reason $\pi_{n}(Y, *)$ may not be a free $\pi$-module is that $\pi_{n} Y$ is not a free $Z$-module. From the above we draw the following

Corollary 1.2. Let $Y=X \vee S$ where $X$ is simply connected and $S$ is one dimensional $\left(\sim\right.$ wedge of circles!). Then one has $\cap_{k=1}^{\infty} I^{k} \pi_{n}(Y, *) \approx 0(n \geqslant 2)$.

Here $I$ denotes the augmentation ideal of the group ring $Z\left[\pi_{1} S\right]$ of the free group $\pi_{1} S$. The filtration of $\pi_{n}$ by powers of the augmentation ideal has

Received by the editors August 1, 1977 and, in revised form, January 12, 1978.

AMS (MOS) subject classifications (1970). Primary 55D15.

'Partially supported by an NSF grant. 
proven to be an important tool for homology theory of non-simply-connected spaces. Corollary 2 implies that all the homotopy groups of $X \vee S$ are residually nilpotent.

2. Proof of Theorem 1. The main idea is to decompose $\pi_{n} Y$ into a direct sum $\bigoplus_{L} B_{L}$ where $L=\left\{g_{1}, \ldots, g_{k}\right\} \subseteq \pi$ in such a way that the natural action of $\pi$ will freely permute the $B_{L}$ 's among themselves. First observe that the universal cover of $X \vee K(\pi, 1)$ is homotopy equivalent to a bouquet $\bigvee_{g \in \pi} X_{g}$ where the action of $g_{1} \in \pi$ sends the copy $X_{g}$ of $X$ to $X_{\left(g \cdot g_{1}\right)}$ by the "identity" map. Intuitively, the direct summand $B_{L}$ of

$$
\pi_{n} Y=\pi_{n}\left(\bigvee_{g \in \pi} X_{g}\right)
$$

is the subgroup of all elements in the group of $X_{L}=\bigvee_{g \in L} X_{g}$ which "depend essentially" on all the $k$ components of $X_{L}$ (where $L=\left\{g_{1} \cdots g_{k}\right\} \subseteq \pi$ ) i.e. which go to zero in all the nontrivial projections $X_{L} \rightarrow X_{L^{\prime}}$ for $L^{\prime} \subset L$; $L^{\prime} \neq L$. Such a decomposition was essentially accomplished (in the finite case) by Eilenberg and Mac Lane [E-M] using the notion of cross-effects, so here we briefly recapitulate their results in our infinite context.

LEMMA 2.1. Let $F$ be a compactly generated functor from pointed spaces to abelian groups and let $S$ be a set. There is a natural decomposition

$$
F\left(\bigvee_{\alpha \in S} X_{\alpha}\right)=\sum_{\left(s_{1}, \ldots, s_{i}\right) \subseteq S} F^{i}\left(X_{s_{1}}|\cdots| X_{s_{i}}\right)
$$

The direct sum $\Sigma$ ranges over all (unordered) finite subsets of $S$ and $F^{i}$ are functors on unordered $i$-tuples of pointed spaces with $F^{i}\left(*\left|X_{1}\right| \cdots \mid X_{i-1}\right)=$ 0 .

Proof. Compare [E-M]. Let $A_{n}$ be the subgroup of $F\left(\bigvee_{\alpha \in S} X_{\alpha}\right)$ generated by all subgroups

$$
A_{J}=\operatorname{Im}\left(F\left(\bigvee_{j \in J} X_{j}\right) \rightarrow F\left(\bigvee X_{\alpha}\right)\right)
$$

where the map is induced by the natural inclusion of $J \subseteq S$ for a finite subset $|J|=n$. We shall prove by induction on $n$ that

$$
A_{n}=\sum_{|L|<n} B_{L}=\sum_{k=|L|<n} F^{|k|}\left(X_{l_{1}}|\cdots| X_{l_{k}}\right)
$$

where $L=\left\{l_{1}, \ldots, l_{k}\right\} \subseteq S, k \leqslant n$, and $B_{L}=F^{k}\left(X_{l_{1}}|\cdots| X_{l_{k}}\right)$ is a natural direct summand assigned to each such $L$. The statement is trivial for $n=0$ and assume it is true for integers less than $n+1$. First we note that $A_{n}$ is a direct summand of $A_{n+1}$. To see this one constructs for the natural inclusion $A_{n} \hookrightarrow A_{n+1}$ a left inverse which is a projection $p: A_{n+1} \rightarrow A_{n}$ : Since $A_{n}$ decomposes by induction into $\bigoplus_{|L| \leqslant n} B_{L}$ and since $F$ is compactly generated it is enough to build a left inverse $p_{L}$ to $B_{L} \subset A_{n} \subset A_{n+1}$ for all $L$ since $\bigoplus p_{L}$ will be well defined ( $\alpha \in A_{n+1} \Rightarrow p_{L}(\alpha) \neq 0$ for only finitely many $L$ 's).

So take $p_{L}$ to be the composition 


$$
p_{L}: A_{n+1} \hookrightarrow F(\bigvee X) \stackrel{\text { proj }}{\rightarrow} F\left(X_{L}\right) \rightarrow F^{|L|}\left(X_{L}\right)
$$

where $F\left(X_{L}\right)=F\left(X_{l_{n}} \vee \cdots \vee X_{l_{k}}\right)$ and the last projection is well defined by the inductive assumption since $|L| \leqslant n$ so that

$$
F\left(X_{L}\right)=F^{|L|}\left(X_{L}\right) \oplus \ldots
$$

Clearly $\bigoplus_{L} p_{L}$ is a left inverse to the inclusion and we can conclude

$$
A_{n+1}(Y)=A_{n}(Y) \oplus R_{n+1}(Y),
$$

where $R_{n+1}$ is natural in $Y$. Now we can define

$$
B_{L}=F^{n+1}\left(X_{l_{n}}|\cdots| X_{l_{n+1}}\right) \stackrel{\text { def }}{=} R_{n+1}\left(X_{l_{1}} \vee \cdots \vee X_{l_{n+1}}\right)
$$

for $|K|=n+1$. It is easy to see that if $K \neq K^{\prime}$ and $|K|=\left|K^{\prime}\right|=n+1$ then $B_{K} \cap B_{K^{\prime}}=0$. Therefore

$$
R_{n+1}(Y)=\bigoplus_{|K|=n+1} B_{K} .
$$

This completes the proof of the decomposition for $A_{n+1}$ and thus the proof of 1.3.

Let us now use 2.1 to prove 1.1. Let

$$
T^{n} Y=\bigoplus_{\left\{l_{1} \cdots l_{n}\right\} \subseteq \pi} F^{n}\left(X_{l_{1}}|\cdots| X_{l_{n}}\right),
$$

where $\pi$ takes the place of $S$ in the lemma, and $\left\{l_{1} \cdots l_{n}\right\}$ runs over all the subsets of $\pi$ with precisely $n$ elements.

Since $\pi$ acts on $\tilde{Y} \simeq \bigvee_{g \in \pi} X_{g}$ by permuting the summands, $T^{n} Y$ is a direct summand of $F(Y)$ as a $\pi$-module since it is clearly closed under the $\pi$-action. So $F(Y)=\bigoplus_{n=1}^{\infty} T^{n} Y$ is a direct sum decomposition of $\pi$-modules. Therefore it is enough to show that $T^{n} Y$ is an induced $\pi$-module for every $n$.

To see this it is enough to show that $\pi$ acts freely on the indexing set of the direct sum decomposition (2.2). But $\pi$ acts freely on itself and since it is torsion-free it also acts freely on the set of its finite subsets, as can easily be seen. Q.E.D.

3. Proof of 1.2. Our 1.2 above follows immediately from 1.1 and the following algebraic lemma.

LeMma 3.1. Let $D$ be an induced $\pi$-module where $\pi$ is a free group. Then $\cap_{k=1}^{\infty} I^{k} D=0$.

The lemma is well known for a free module over a free group.

Proof. Let $A \otimes_{\mathrm{Z}} B=A \otimes B$ denote the tensor product over the integers Z. We first make the following observation: If $D=Z_{\pi} \otimes M$ then $I^{k} D=I^{k}$ $\otimes M$. To see this observe that tensoring the short exact sequence

$$
0 \rightarrow I \rightarrow \mathbf{Z} \pi \rightarrow \mathbf{Z} \rightarrow 0
$$

with $D$ over $\pi$ preserve exactness since it is the same as tensoring with $M$ over $\mathbf{Z}$, but the sequence splits over $\mathbf{Z}$ ! (Alternatively $\operatorname{Tor}_{i}^{\pi}(D, \mathbf{Z})=0$ for $i>0$ compare [C-E].) Thus we get a short exact sequence 


$$
0 \rightarrow I \otimes M \rightarrow D \rightarrow D \otimes_{\pi} \mathbf{Z} \rightarrow 0
$$

which means precisely that $I D \approx I \otimes_{\pi} D \approx I \otimes M$. Now since for a free group $\pi$ all the powers of the augmentation ideal are free $\pi$-modules, they are induced, so we can use the same equations as above to get by induction on $k$ :

$$
\begin{aligned}
I^{k+1} D & =I\left(I^{k} \otimes M\right)=I \otimes_{\pi}\left(I^{k} \otimes M\right) \\
& \approx\left(I \otimes_{\pi} I^{k}\right) \otimes M \approx I^{k+1} \otimes M .
\end{aligned}
$$

Thus one must show $\cap_{k} I^{k} \otimes M=0$. Ignoring the $\pi$-structure of these abelian groups we note that

$$
0 \rightarrow I^{k+1} \rightarrow I^{k} \rightarrow I^{k} / I^{k+1} \rightarrow 0
$$

is a $\mathbf{Z}$-split exact sequence of free abelian groups with $\cap I^{k}=0$ (compare [F]). This readily implies the result since if $b_{k}$ are chosen bases for the free abelian groups $I^{k}$ with $b_{k+1} \subset b_{k}$ we have

$$
\bigcap_{k}\left(I^{K} \otimes M\right)=\bigcap\left(\bigoplus_{b_{k}} M\right)=\bigoplus_{b} M
$$

where $b=\cap b_{k}=\varnothing$.

\section{REFERENCES}

[B-D] I. Bernstein and E. Dror, On the nomotopy type of non-simply connected co-H-space, Illinois J. Math. 20 (1976), 528-534.

[E-M] S. Eilenberg and S. Mac Lane, On the groups $K(\pi, n)$. II, Ann. of Math. (2) 60 (1954), 49-139.

[F] R. H. Fox, Free differential calculus. I, Ann. of Math. (2) 57 (1953), 547-549.

[G] T. Ganea, Some problems on numerical homotopy invariants, Lecture Notes in Math., vol. 249, Springer-Verlag, Berlin and New York, pp. 23-30.

[HMR] P. Hilton, G. Mislin and J. Roitberg, On co-H-spaces (preprint).

[C-E] H. Cartan and S. Eilenberg, Homological algebra, Princeton Univ. Press, Princeton, N.J., 1956.

Department of Mathematics, Cornell University, Ithaca, New York 14853

Department of Mathematics, Hebrew University of Jerusalem, Jerusalem, Israel 